Referencia para citar este artículo: Sola-Morales, S., \& Campos-Garrido, N. A. (2019). Discurso estatal chileno en la protección de los derechos de niños(as) y jóvenes. Revista Latinoamericana de Ciencias Sociales, Niñez y Juventud, 17(1), 105-124. doi:https://dx.doi.org/10.11600/1692715x.17106

\title{
Discurso estatal chileno en la protección de los derechos de niños(as) y jóvenes*
}

\author{
SALOMÉ SOLA-MORALES M* $^{*}$ \\ Profesora Universidad de Sevilla, España. \\ Nicole Alejandra Campos Garrido ${ }^{* * * *}$ \\ Periodista, Chile.
}

\section{Artículo recibido en noviembre 11 de 2017; artículo aceptado en enero 1 de 2018 (Eds.)}

- Resumen (analítico): el objetivo de esta investigación ha sido analizar el discurso estatal chileno en la protección de los derechos de niños, niñas y de los y las jóvenes. Para ello se ha utilizado una metodología cuantitativa y cualitativa utilizando centrada en el análisis del discurso del Estado. El principal resultado ha sido que las y los menores de 18 años desempeñan roles secundarios en las políticas estatales y no son considerados actores sociales en el discurso del Estado chileno sobre infancia y juventud. Además, no expresan opiniones y son discriminados cuando pertenecen a un contexto social precario o han infringido la ley. Como conclusión, se puede afirmar que el discurso del Estado chileno se opone a la propuesta de la Convención de los Derechos del Niño (y la niña), firmada el 20 de Noviembre 1990.

Palabras clave: Chile, discurso, Estado, infancia, juventud, participación (Tesauro de Ciencias Sociales de la Unesco).

\section{Discourse of the Chilean state the protection of children and young people's rights}

- Abstract (analytical): The objective of this research has been to analyze the Chilean state's discourse on the protection of children and young people's rights. We used quantitative and qualitative methodologies to carry out this research that were focused on an analysis of the discourse of the Chilean state. The main finding is that young people under the age of 18 play secondary roles in government policies and are not considered social actors in the Chilean state's discourse on children and young people. In addition, they don't or can't express opinions and they are discriminated against when they form part of a precarious social context or they have broken the law. In conclusion, the

\footnotetext{
Este artículo de investigación científica y tecnológica presenta resultados de la investigación denominada «Discurso del Estado sobre Infancia y Juventud durante los Gobiernos de Michelle Bachelet (2006-2010) y Sebastián Piñera (2010-2014)», presentada por Nicole Alejandra CamposGarrido, dirigida por la Dra. Salomé Sola-Morales, Universidad de Santiago de Chile, 2015. Acta número: 31102015. Inicio de la investigación: 1/03/2013. Finalización: 31/05/2015. Área: Ciencias Sociales. Sociología. Subárea: Temas Sociales.

** Doctora en Medios, Comunicación y Cultura por la Universidad Autónoma de Barcelona, España. Magíster en Política y Democracia, de la Universidad Nacional de Educación a Distancia, España. Profesora Universidad de Sevilla. Miembro de Grupo Interdisciplinario de Estudios en Comunicación, Política y Cambio Social, Compolíticas. Universidad de Sevilla. Orcid: https://orcid.org/0000-0001-7085-4595. Índice H5: 4. Correo electrónico: ssolamorales@gmail.com

*** Periodista y Magister en Ciencias de la Comunicación de la Universidad de Santiago de Chile. Correo electrónico: nicolecamposgarrido@gmail.com
} 
study affirms that the discourse of the Chilean state goes against the principles of the United Nations Convention on the Rights of the Child, signed on the 20th of November 1990.

Key words: Chile, discourse, State, childhood, youth, participation (Social Sciences Unesco Thesaurus).

\section{Discurso do Estado chileno sobre a proteção dos direitos das crianças e dos jovens}

- Resumo (analítico): o objetivo desta pesquisa foi analisar o discurso do Estado chileno na proteção dos direitos das crianças e dos jovens. Para tanto, utilizou-se uma metodologia quantitativa e qualitativa, focada na análise do discurso do Estado. A principal descoberta foi que os menores de 18 anos desempenham papéis secundários e não são considerados atores sociais no discurso do Estado chileno sobre infância e juventude. Além disso, eles não expressam opiniões e são discriminados quando pertencem a um contexto social precário ou violaram a lei. Em conclusão, pode-se afirmar que o discurso do Estado chileno se opõe à proposta da Convenção sobre os Direitos da Criança, assinada 20 Novembro de 1990.

Palavras-chave: Chile, discurso, Estado, infância, juventude, participação (Thesaurus de Ciências Sociais da Unesco).

\section{-1. Introducción. -2. Objetivos y preguntas de investigación. -3. Metodología. -4. Resultados. -5 . Conclusiones. -Lista de referencias.}

\section{Introducción}

La infancia y la juventud son construcciones simbólicas y sociales que han ido cambiando a lo largo del tiempo, en función del contexto en el que se desarrollan (James \& Prout, 1990). Por consiguiente, son atravesadas por prácticas culturales, sociales y políticas, que no solo definen las formas de pensar en niñas y niños y los y las jóvenes, sino también en cómo ellos y ellas se entenderán a sí mismos/as y entre sí (Marín, 2011). Tales construcciones simbólicas generan interacciones que limitan «la posibilidad de experiencias o perspectivas de análisis fuera de esta lógica» (Casas, 2006, p. 30).

La infancia, ha sido muchas veces circunscrita a una condición de instrumento para explicar las claves del mundo adulto (Gaitán, 2006; Rodríguez, 2007), ya sea entendiéndola como tabula rasa que se puede moldear fácilmente debido a su inherente pasividad y subordinación, como diría John Locke (citado en Rice, 1997), o como una etapa pre-social, de preparación para la integración a la sociedad (mundo adulto) que ocurre precisamente cuando las personas abandonan su condición infantil (Berger \& Luckmann, 1968; Durkheim, 1975). Hoy en día la nueva sociología de la infancia, por el contrario, reivindica al niño o a la niña como agente social, cuya capacidad y creatividad es determinante en el proceso de construcción de las relaciones sociales y culturales. Es decir, la infancia es considerada como un fenómeno social, no pre-social o asocial (Rodríguez, 2007).

En Chile, en las últimas décadas, la construcción de infancia se ha visto transformada por la suscripción del Estado chileno a la Convención de los Derechos del Niño (y la niña) a comienzos de los años 90 (Organización de las Naciones Unidas-ONU, 1989). Integrar este acuerdo internacional a las normativas nacionales vigentes ha influenciado la forma de hacer política para este segmento de la población. El cambio desde la Doctrina de la Situación Irregular (1928) a la Protección Integral (1990), ha provocado una serie de reformas en el aparato jurídico, desarrollándose leyes penales, de familia, educacionales e, incluso, artículos en la Ley de Prensa (República de Chile, 2001), que definen cómo esta última debe tratar a las personas menores de 18 años.

Estas transformaciones han afectado la institucionalidad y también las perspectivas que tiene la sociedad chilena sobre niñas y niños, y los y las jóvenes, creándose discursos que no solo están asociados al rango etario, sino también a aspectos económicos, sociales y culturales. 
En paralelo a las instituciones de infancia y juventud existentes (Junji, Integra, Sename e Injuv, que serán explicadas más adelante), ha surgido en marzo del 2014 el Consejo de la Infancia, instancia asesora de la presidencia, que pretende generar una nueva Ley de Garantía Universal de Derechos de la Niñez, para construir un marco legal que guíe al Estado en el cumplimiento de la Convención de los Derechos del Niño (y la niña).

No obstante, Giorgi (2012) y Villagrasa (2015) advierten que este nuevo paradigma no ha logrado imponerse. La doctrina de situación irregular aún subyace tanto en situaciones cotidianas como dentro de las instituciones responsables de planificar y ejecutar las políticas de infancia. Un ejemplo de lo anterior se encuentra en la Ley 16.618 de menores (República de Chile, 1967) la que todavía, de acuerdo con Contreras, Rojas y Contreras (2015) «presenta una terminología inadecuada, y conceptos desactualizados (que datan de los años sesenta), lo cual refleja dificultades fundamentalmente conceptuales en el abordaje de los temas de infancia» (p. 99). Del mismo modo, Durán-Strauch (2017), evidencia que ambos discursos coexisten, y que «las perspectivas que se han llamado de situación irregular y de Derechos de niños y niñas: protección integral no son casillas opuestas y excluyentes sino extremos de un continuum en el cual se ubican las diferentes posiciones frente al tema» (p. 884).

En el aspecto social, en los últimos años han aumentado los espacios para la defensa de la infancia y la juventud, tanto desde el Estado como desde la sociedad civil, y si bien siguen existiendo importantes falencias, sí que se ha comenzado a poner en discusión la ausencia de una perspectiva de derechos. En este sentido, el desafío de los sistemas políticos y jurídicos es que se les reconozca al niño y a la niña y a los y las jóvenes la titularidad de sus derechos. Ello quiere decir: por un lado, que los puedan ejercer directamente, sin tener que acudir a intermediarios/as como madres, padres o representantes legales y, por otro, que no se les limite al ejercicio de los mismos en la medida de la evolución de sus facultades (Lozano-Vicente, 2016). En otras palabras, «reconocer una niñez portadora de derechos que debe ser tratada con plena consideración y respeto, no solo en términos simbólicos sino también legales, garantizando espacios jurídicos y políticos para su participación activa en las decisiones que la involucran» (Díaz-Bórquez, Contreras-Shats, \& Bozo-Carrillo, 2018, p. 103). Para lograrlo será necesario resignificar lo que entendemos por infancia, eliminando del lenguaje «la relación dicotómica y antagónica, una mirada política del sujeto donde no haya menores ni mayores, superiores o inferiores, débiles o fuertes» (Arias, 2017, p. 131).

En razón a lo expuesto se ha estudiado el discurso reproducido por una serie de instituciones que trabajan directamente con la población infanto-juvenil entre 2006 y 2013, construyendo una aproximación teórica sobre discurso del Estado chileno en la protección de los derechos sobre infancia y juventud. Las variables y categorías principales del estudio han sido: las estrategias discursivas utilizadas por el Estado, actores presentes y ausentes en el discurso estatal, papel que representan los niños y las niñas, y también los y las jóvenes; rol, acción que realizan y perspectiva de derechos.

\section{Objetivos y preguntas de investigación}

El objetivo principal de esta investigación es analizar el discurso del Estado chileno en torno a infancia y juventud a través de sus principales instituciones. Para ello se han desarrollado los siguientes dos objetivos específicos:

- Explorar cuáles son los elementos que componen la propuesta discursiva desde las instituciones que trabajan con infancia y juventud.

- Comparar el discurso de Junji, Integra, Sename e Injuv durante los gobiernos de Michelle Bachelet y el gobierno de Sebastián Piñera. Con el fin de establecer si acaso existen diferencias y similitudes discursivas entre un gobierno de centro-izquierda y otro de centro-derecha. 
Las preguntas que han guiado esta investigación son las siguientes:

P1: ¿Cómo es el discurso del Estado chileno en torno a infancia y juventud?

P2: ¿Cuáles son los elementos clave que componen la propuesta discursiva desde las instituciones que trabajan con infancia y juventud?

P3: ¿Existen diferencias y similitudes en torno al discurso sobre infancia y juventud desarrollado por Junji, Integra, Sename e Injuv entre el gobierno de Michelle Bachelet y el de Sebastián Piñera?

\section{Metodología}

Se ha realizado un estudio explicativo, cuantitativo y cualitativo (mixto) y de carácter no experimental (ex post facto). La técnica empleada para la recolección de información fue la observación de fuentes documentales, a través del análisis de contenido y de discurso.

\subsection{Muestra}

La muestra fue construida atendiendo al criterio de selección de periodicidad de 8 años y al criterio temático de dos gobiernos completos. Se tomaron todos los balances de gestión publicados en este período. Se trata de un total de 32 balances de gestión publicados desde el año 2006 hasta el 2014, es decir, durante los gobiernos de Michelle Bachelet (marzo del 2006 a marzo del 2010) y el de Sebastián Piñera (marzo de 2010 a marzo de 2014). No se ha incluido el balance de gestión correspondiente al año 2014 porque estos evaluaron el trabajo realizado durante el primer año del segundo gobierno de Michelle Bachelet, quien asumió el cargo el 11 de marzo del 2014.

Las instituciones fueron seleccionadas bajo la premisa de que todas debían trabajar directamente con la población infantojuvenil, considerando para esta investigación las cuatro más relevantes del Estado chileno:

1. Servicio Nacional de Menores (Sename): organismo gubernamental centralizado, colaborador del sistema judicial y dependiente del Ministerio de Justicia. Se encarga de la protección de derechos de niños, niñas y adolescentes, y de las y los jóvenes entre 14 y 18 años ante el sistema judicial. Además, se ocupa de regular y controlar la adopción en Chile.

2. Fundación Integra: es uno de los principales prestadores de educación parvularia en Chile, trabaja con niños y niñas entre 84 días y 3 años 11 meses y 29 días de edad que se encuentran en situación de pobreza y/o vulnerabilidad.

3. Junta Nacional de Jardines Infantiles (Junji): es una institución del Estado de Chile creada en 1970 por la Ley $\mathrm{N}^{\circ}$ 17.301, como un estamento autónomo vinculado al Ministerio de Educación y cuyo fin es atender la educación inicial del país. Su compromiso consiste en entregar educación parvularia a niños y niñas, preferentemente menores de cuatro años y en situación de vulnerabilidad social.

4. Instituto Nacional de la Juventud (Injuv): es un organismo de servicio público encargado de colaborar con el Poder Ejecutivo en el diseño, planificación y coordinación de las políticas relativas a los asuntos juveniles. Orienta su trabajo a los y las jóvenes entre 15 y 29 años.

\subsection{Técnica de recolección de datos e instrumentos de análisis}

La técnica empleada para la recolección de datos fue la observación y análisis de fuentes documentales. La metodología utilizada fue el análisis de contenido y discurso. Para ello se diseñó un instrumento que cuenta con variables cuantitativas y cualitativas, ficha de análisis, y un libro de códigos que acompaña y explica el proceso para la recolección de datos. Este fue desarrollado con el 
fin de unificar criterios y disminuir la posibilidad de que los hallazgos presentaran sesgos. La ficha se ha elaborado atendiendo a alguna de las siguientes propuestas: Análisis Crítico del Discurso (ACD), Análisis de Contenido y Teoría del Framing.

\subsubsection{Análisis Crítico del Discurso (ACD)}

Del ACD se han tomado como referencia las propuestas metodológicas de Van Dijk (2009), poniendo énfasis en tres aspectos:

- Los elementos pragmáticos, prescriptivos y narrativos, presentes en la identificación de proposiciones en los balances de gestión que demuestran las acciones del Estado y sus enfoques en el momento de hablar sobre infancia y juventud.

- Los elementos persuasivos, que en este caso serían las explicaciones propuestas por las instituciones y su tipología, que establecen las bases argumentales del discurso sobre infancia y juventud.

- La visión dimensional del poder que responde a las instituciones que lo conforman, la cual ha ayudado a crear el instrumento que evidencia las propuestas discursivas del Estado.

\subsubsection{Análisis de Contenido}

Del Análisis de Contenido se ha rescatado la identificación de tendencias en el discurso, puesto que es «una técnica de investigación para la descripción objetiva, sistemática y cuantitativa del contenido manifiesto de las comunicaciones con el fin de interpretarlas» (Berelson, 1971, p. 18).

\subsubsection{Teoría del Framing}

También se ha contemplado la teoría del Framing, concretamente en las ideas de Tankard (1991), según las cuales el discurso se convierte en «la idea central organizadora del contenido que aporta un contexto mediante un proceso de selección, énfasis, exclusión y elaboración» (p. 5). Así ha sido posible observar a los actores y fuentes que son incluidos en los relatos del Estado, así como aquellos participantes y voces excluidas de los textos analizados.

\section{Resultados}

El análisis comparado realizado ha permitido constatar cuáles son los elementos que componen la propuesta discursiva de las instituciones seleccionadas. Con el fin de conocer cómo es el discurso y el rol del Estado en relación con infancia y juventud se han explorado los siguientes ítems:

1. Definición del «ellos» y delimitación de los grupos externos.

2. Identificación de niñas y niños, y los y las jóvenes.

3. Perspectiva de género.

4. Acciones realizadas por las niñas y niños, y los y las jóvenes.

5. Actores ausentes en el discurso.

6. Auto-representación del «nosotros», en este caso, el Estado.

7. Acciones realizadas por el Estado por periodo de gobierno.

8. Estrategias discursivas.

9. Rol del estado. 


\subsection{Definición del «ellos» y delimitación de los grupos externos}

En los balances de gestión analizados se ha podido observar cómo el Estado define el «nosotros» y lo utiliza como parámetro en la construcción del «ellos», estableciendo diferencias, clasificaciones y valoraciones. La construcción de la otredad resulta clave para entender el discurso estatal ya que muestra la realidad desde la que se habla y permite describir la perspectiva que las distintas organizaciones tienen sobre infancia y juventud. Además de mostrar su relación con otros grupos relacionados como madres, padres, apoderados y organizaciones sociales.

La construcción del «ellos»o de los «otros» se presenta en el corpus analizado de múltiples formas: en relación con la importancia otorgada a los grupos externos para el desarrollo de políticas, en la participación que estos tienen en el relato y en cómo son nombrados y descritos. El grupo externo principal en relación con las organizaciones estatales está integrado por niñas y niños, y los y las jóvenes, del que se desprenden otros relacionados como madres, padres y/o familia. Un segundo grupo externo está compuesto por las organizaciones que trabajan con niñas y niños, y con los y las jóvenes pero que no pertenecen a la institucionalidad estatal.

Tabla 1. Grupos externos

\begin{tabular}{|c|c|c|}
\hline & Tipo de grupo & Grupo \\
\hline \multirow{4}{*}{$\begin{array}{l}\text { «Ellos» o los } \\
\text { «otros» }\end{array}$} & \multirow{4}{*}{ Externo } & Niñas, niños y/o jóvenes \\
\hline & & Madres, padres y/o familias \\
\hline & & ONG que trabajan con infancia y/o juventud \\
\hline & & $\begin{array}{l}\text { Otras organizaciones que trabajan con infancia y/o } \\
\text { juventud }\end{array}$ \\
\hline
\end{tabular}

El uso de la palabra «clientes», para describir a las personas que utilizan los servicios o integran los programas, estuvo presente en todos los balances de gestión revisados, sin existir una distinción entre gobiernos o instituciones. Esta palabra remite al imaginario empresarial que se ha instaurado en las organizaciones estatales en las últimas décadas. Al preferir este término frente a otros se destaca la naturaleza de un Estado asistencialista y neoliberal, a cuyos métodos de intervención, como señala Grassi (2003, p. 6), se le atribuyen un nivel de dependencia y/o cierto grado de subordinación política. Es decir que «sitúa a las personas como objetos de intervención [...] sin capacidad de ser parte e incidir en los diferentes momentos de la misma» (Valverde, 2008, p. 111).

Se puede observar que dos de las cuatro instituciones públicas analizadas (Junji y Sename), proponen sus objetivos estratégicos en primera persona, es decir emplazan la acción en la institución y no en la importancia que esta podría tener para el desarrollo de niñas y niños, y los y las jóvenes. De esta forma, el énfasis discursivo se mantiene en la organización. Un ejemplo, es el objetivo estratégico presentado en el Balance de gestión de Sename (2011, p. 3): «Desarrollar y optimizar la oferta de programas que contribuyan al sistema de protección social de la infancia y adolescencia».

Si se desglosa el objetivo, se puede inferir que los verbos «desarrollar y optimizar» contribuyen al «sistema de protección social de infancia y adolescencia», es decir, a mejorar un programa de Sename. Por lo tanto, no está explícita la intención de mejorar la situación que viven niños y niñas, y los y las jóvenes atendidos en sus centros. La forma en que se plantean los objetivos demuestra que el énfasis está en el quehacer del organismo y no en sus beneficiarios/as.

\subsection{Identificación de niñas y niños, y los y las jóvenes}

Otro aspecto asociado a la representación de «los otros» por parte del Estado, es la identificación de niños y niñas, y los y las jóvenes, es decir, cómo son mencionadas las personas menores de 18 años en los textos. 
Gráfico 1. Identificación de niñas y niños, y los y las jóvenes

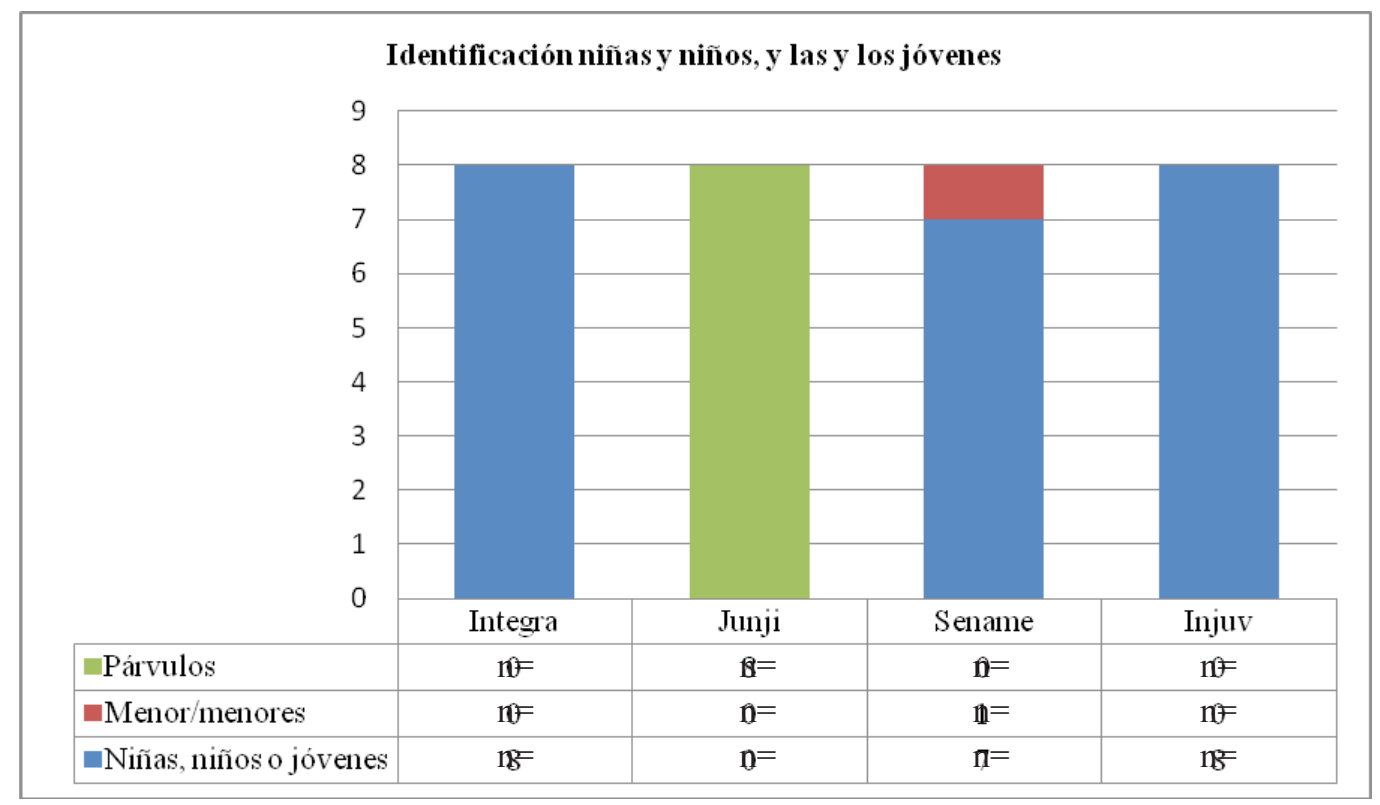

En Integra y Junji existen diferencias al nombrarlos/las. Mientras la primera se refiere a «niñas y niños», otorgándoles plenos derechos como sujetos y ciudadanos, Junji recurre al uso de la palabra «párvulos». De acuerdo con la Real Academia Española (RAE), párvulo tiene tres acepciones: «dicho de un niño, de muy corta edad»; «inocente, que sabe poco o es fácil de engañar»y «humilde, cuitado» (2001, párr. 1, 3, 4). Podemos observar que en los últimos significados, el concepto «párvulo» define a niñas y niños con términos como iluso/a (concepto que se desprende de fácil de engañar), apenados/ as, afligidos/as, apocados/as, (términos que se desprenden de humilde y cuitado). Lo que reafirma la noción de la infancia como objeto de protección y asistencialismo. Sename, por otra parte, fue la única institución que utilizó en una oportunidad la palabra «menor» para referirse a niñas y niños, y a los y las jóvenes. Término peyorativo que significa «menos importante con relación a algo del mismo género» (RAE, 2001, párr. 2). Este concepto remite a la doctrina de la situación irregular que ve a niñas y niños como «seres caracterizados por su incapacidad y su fragilidad. Esta concepción implica, según los casos, que sean objeto de protección y de asistencia, u objeto de represión» (Gülgönen, 2016, p. 83). Tiene una significación negativa que implica «reducción, limitación e incapacidad» (Misle \& Pereira, 2003, p. 2). «Se utiliza como un sello para describir la condición social de niños, niñas y de los y las jóvenes que no cuentan con oportunidades, por lo que también es asociado a pobreza y criminalidad» (Misle \& Pereira, 2003, p. 3).

\subsection{Perspectiva de género}

Con respecto a la perspectiva de género, se pudo notar un cambio entre los informes realizados en ambos periodos, destacando el gobierno de Bachelet al entablar una simetría entre la población femenina y masculina en los planteamientos de las diferentes instituciones, reflejada en el uso de «los y las jóvenes» para el caso de Injuv, y «niñas y niños» cuando se trata de Sename, Junji o Integra. Por su parte, las instituciones bajo el mandato de Sebastián Piñera solo mantuvieron el uso de palabras inclusivas en su misión, la que no varió si se compara con el gobierno que le antecede. 


\subsection{Aciones realizadas por las niñas y niños, y los y las jóvenes}

Las acciones que se les atribuyen a niñas y niños, y a los y las jóvenes también permiten delimitar y definir quiénes son «los otros/as» y lo que hacen. En Integra, Junji, Sename e Injuv la acción que más se repitió fue participar ( $\mathrm{n}=46)$, como se puede observar en el gráfico 2 .

Gráfico 2. Acciones que realizan niñas y niños y los y las jóvenes

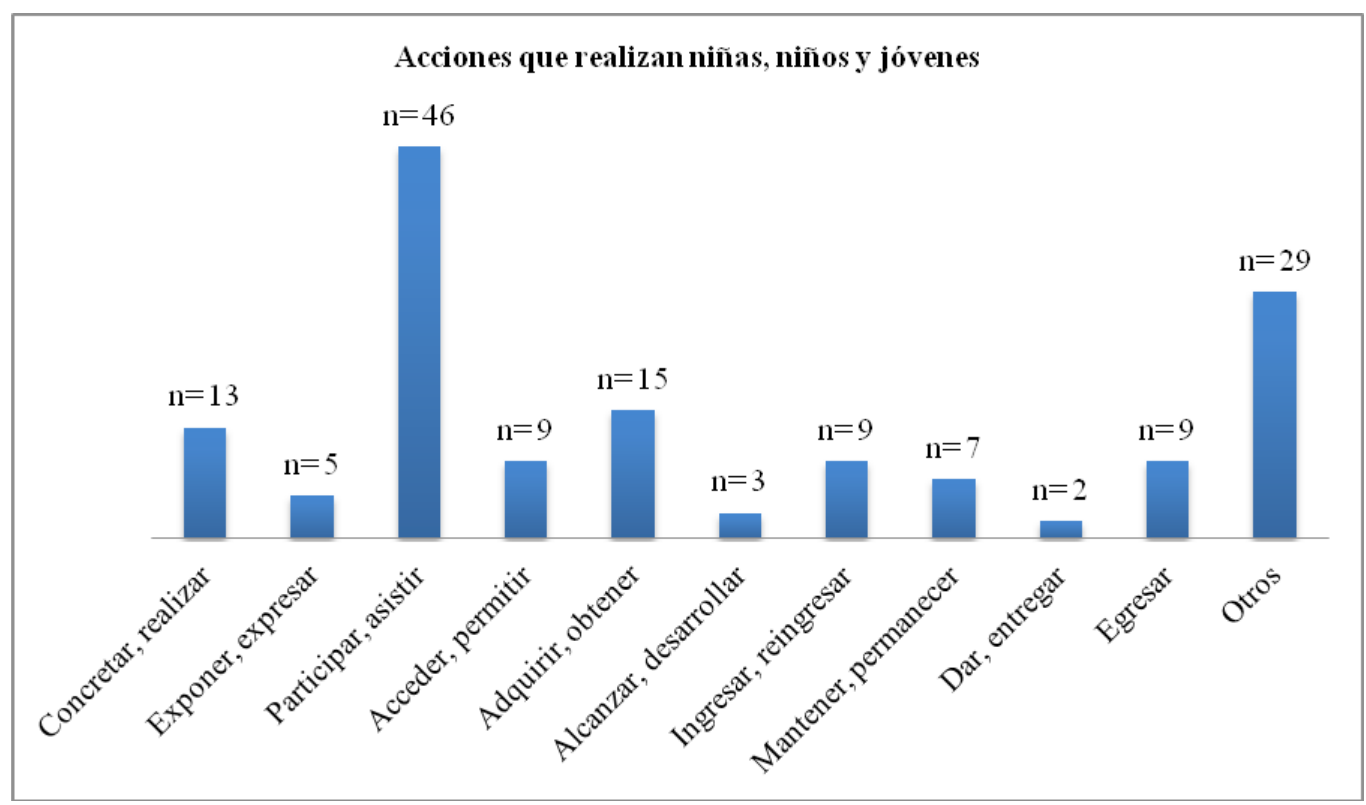

Según el Diccionario de la Real Academia Española (RAE, 22. ${ }^{a}$ edición), el verbo «participar» significa tomar parte en algo; recibir una parte de algo; compartir, tener las mismas opiniones, ideas, etc., que otra persona; dar parte o comunicar (2001, párr. 2, 3). La participación supone que niñas y niños, y las y los jóvenes expresen opiniones y decisiones con respecto a asuntos que les afectan directamente. Hart (1993) afirma que «la participación es la capacidad para expresar decisiones que sean reconocidas por el entorno social y que afectan a la vida propia y/o a la vida de la comunidad en la que uno vive» (p. 5). Sin embargo, se puede observar como la participación propuesta por las instituciones es simbólica, ya que se usa como sinónimo de la palabra «asistir», que significa acompañar, concurrir, estar o hallarse presente, lo que no implica una participación activa o vinculante. De acuerdo con Del Río (2011), para que haya participación real niñas y niños deben ser protagonistas de los procesos y no «beneficiarios de ciertos elementos que, se considera, les aportarán en su desarrollo, pero definidos desde lugares ajenos a ellos mismos» (p. 102).

No obstante, se puede observar que las acciones que denotan participación e inclusión, en el sentido de que requieren de una intervención en un hecho, como «exponer y expresar», están asociadas solo a los y las jóvenes y no a los niños. Si se desglosan las acciones por institución se puede notar que Integra es la que concentra la mayoría de las acciones, sobre todo participar/asistir ( $\mathrm{n}=18)$, acceder/ permitir $(n=8)$, adquirir y obtener $(n=14)$. Mientras que en Junji, los y las jóvenes solo están presentes en las actividades $(n=10)$ o se les menciona en las tasas de ingreso $(n=1)$ y permanencia $(n=1)$. Algo similar ocurre en Sename, que además de asistir y participar $(n=7)$, concurren a los centros ingresan/ reingresan $(n=7)$, permanecen $(n=3)$ y egresan $(n=8)$. En Injuv, además de participar y asistir $(n=$ 11) se encuentran las acciones exponer y expresar $(n=4)$. 
Tabla 2. Acción realizada por niñas y niños, y las y los jóvenes por organización

\begin{tabular}{|c|c|c|c|c|c|c|}
\hline \multicolumn{7}{|c|}{ Acción realizada por niños, niñas, y jóvenes por organización } \\
\hline & & \multicolumn{4}{|c|}{ Organización } & \multirow{2}{*}{ Total } \\
\hline & & Integra & Junji & Sename & Injuv & \\
\hline \multirow{11}{*}{$\begin{array}{l}\text { Acción realizada } \\
\text { por niños, niñas, y } \\
\text { jóvenes }\end{array}$} & Concretar, realizar & 5 & 0 & 3 & 5 & 13 \\
\hline & Exponer, expresar & 0 & 0 & 1 & 4 & 5 \\
\hline & Participar, asistir & 18 & 10 & 7 & 11 & 46 \\
\hline & Acceder, permitir & 8 & 0 & 0 & 1 & 9 \\
\hline & Adquirir, obtener & 14 & 0 & 0 & 1 & 15 \\
\hline & Alcanzar, desarrollar & 3 & 0 & 0 & 0 & 3 \\
\hline & Ingresar, reingresar & 1 & 1 & 7 & 0 & 9 \\
\hline & Mantener, permanecer & 3 & 1 & 3 & 0 & 7 \\
\hline & Dar, entregar & 1 & 0 & 0 & 1 & 2 \\
\hline & Egresar & 1 & 0 & 8 & 0 & 9 \\
\hline & Otros & 20 & 0 & 4 & 5 & 29 \\
\hline \multicolumn{2}{|r|}{ Total } & 74 & 12 & 33 & 28 & 147 \\
\hline
\end{tabular}

\subsection{Actores ausentes en el discurso}

Los actores ausentes son todos aquellos que a pesar de tener injerencia en las temáticas desarrolladas, no son mencionados en el relato. La categoría con más presencia fue «organizaciones relacionadas a educación y primera infancia» $(\mathrm{n}=14)$, seguida de «otras organizaciones del área de juventud» $(\mathrm{n}=8)$ y «Familias» $(\mathrm{n}=7)$. La ausencia predominante se debe a la sumatoria de las dos organizaciones que se dedican a educación pre-escolar: Junji e Integra. 
Tabla 3. Actores ausentes en el discurso del Estado

\begin{tabular}{|c|c|c|c|c|c|c|}
\hline \multicolumn{7}{|c|}{ ¿Qué actor no aparece? } \\
\hline & & \multicolumn{4}{|c|}{ Organización } & Total \\
\hline & & Integra & Junji & Sename & Injuv & \\
\hline \multirow{5}{*}{$\begin{array}{l}\text { ¿Qué } \\
\text { actor no } \\
\text { aparece? }\end{array}$} & Familias & 0 & 0 & 7 & 0 & 7 \\
\hline & $\begin{array}{l}\text { Otras organizaciones de infancia } \\
\text { y juventud }\end{array}$ & 0 & 0 & 1 & 0 & 1 \\
\hline & $\begin{array}{l}\text { Otras organizaciones del área de } \\
\text { juventud }\end{array}$ & 0 & 0 & 0 & 8 & 8 \\
\hline & $\begin{array}{l}\text { Otras organizaciones de } \\
\text { educación y primera infancia }\end{array}$ & 8 & 6 & 0 & 0 & 14 \\
\hline & $\begin{array}{l}\text { Otras organizaciones de infancia } \\
\text { y juventud, incluyendo familias }\end{array}$ & 0 & 2 & 0 & 0 & 2 \\
\hline \multicolumn{2}{|l|}{ Total } & 8 & 8 & 8 & 8 & 32 \\
\hline
\end{tabular}

Al relacionar los actores que no aparecen con las instituciones, se pudo encontrar que las familias no son mencionadas en las evaluaciones realizadas por Sename, y solo en un caso se incluyó a otras organizaciones de infancia y juventud. Ello demuestra que la organización no está involucrando a la familia, en contraposición a lo descrito en el artículo 5 de la Convención de los derechos del niño, que señala: «Es obligación del Estado respetar las responsabilidades y los derechos de los padres y madres, así como de los familiares».

En Integra y Junji, los actores excluidos fueron otras organizaciones de educación y primera infancia. En dos casos Junji, además de relegar a otras organizaciones con las que comparte los mismos temas, también descartó a las familias. En este sentido estas organizaciones destinadas a brindar educación inicial, parecen no estar interesadas en establecer un trabajo conjunto con instituciones civiles que se desenvuelvan en materia de educación, y con ello también imposibilitan el flujo de nuevas ideas o formas para mejorar la enseñanza. Por último, los ausentes en los informes de Injuv fueron otras organizaciones del área de juventud. Si bien se podría decir que las familias no fueron mencionadas en estos casos, resulta ser poco relevante porque el enfoque de Injuv busca promover un mayor grado de independencia en los sectores juveniles.

\subsection{Auto-representación del «nosotros», en este caso, el Estado}

El Estado habla del «nosotros» a través de la reiteración de sus acciones, queriendo destacarse como un ente eficaz en la protección, educación y cuidado de niñas, niños y jóvenes. Estas acciones son presentadas a través de cifras con el objetivo de otorgarle mayor «credibilidad» a sus afirmaciones. Al mismo tiempo, se omiten acciones negativas y pasan por alto cuestionamientos a los que han sido sometidas algunas de las organizaciones. Nunca se habla de resultados, de esta forma evita mencionar los casos en los que programas de intervención, campañas o modelos implementados no han dado los 
frutos esperados. En definitiva, hay una omisión de resultados y acciones negativas.

Las acciones que predominan son «realizar, hacer» $(n=141)$, seguidas por «dar» $(n=99$ casos $)$, $\mathrm{y}$ «fortalecer» $(\mathrm{n}=73)$. Todas estas estas acciones pretenden reafirmar la idea de que el Estado hace su trabajo. La acción «dar» está relacionada a los beneficios que entregan las diferentes entidades, reforzando la imagen de un Estado benefactor, y por tanto paternalista.

Gráfico 3. Acciones realizadas por las instituciones estatales

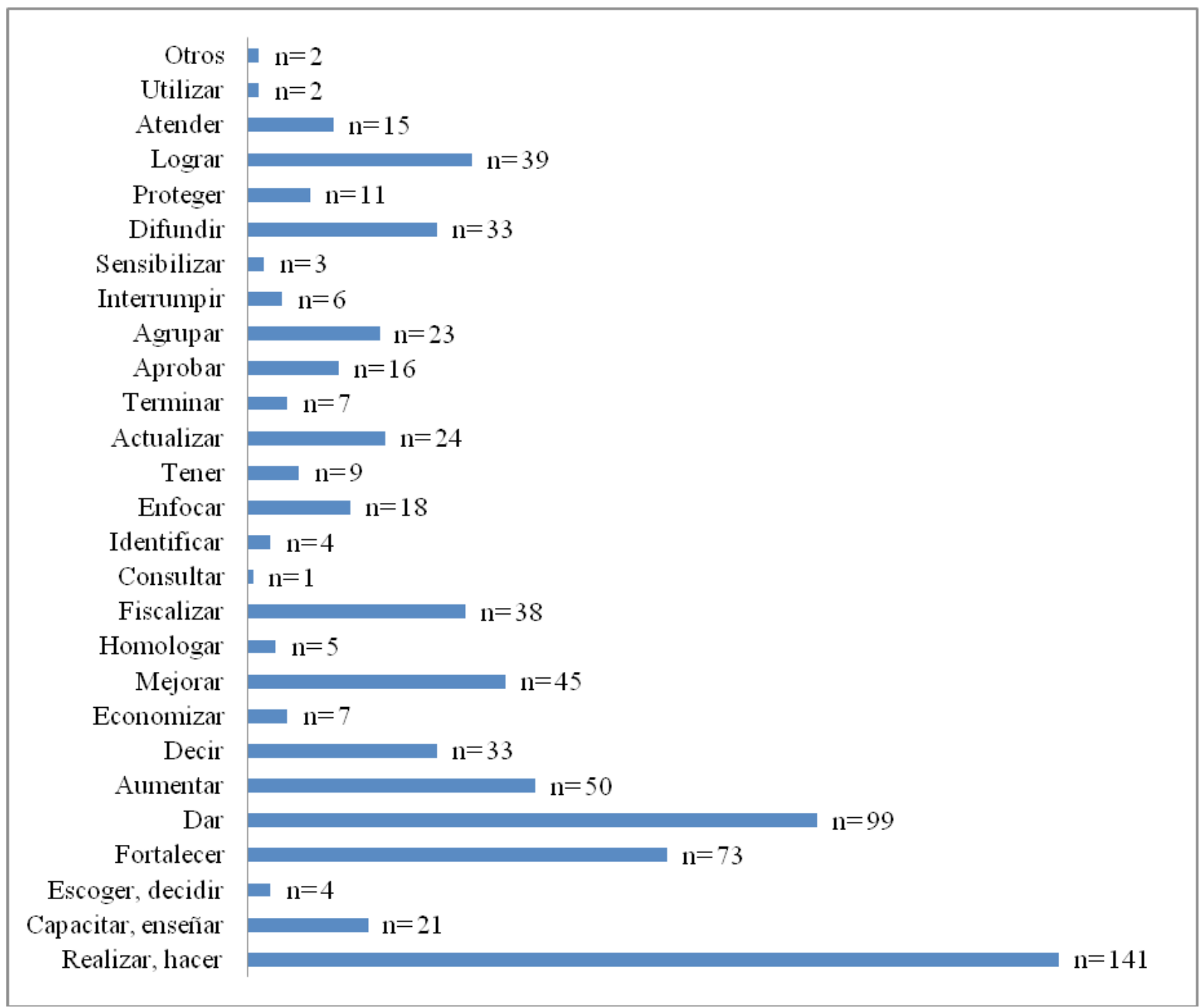

Si se desglosan las acciones por institución, se puede advertir que la categoría «realizar, hacer» es la que predomina en Integra, Junji, Sename e Injuv $(n=141)$. En tanto «dar», aparece en un segundo lugar siguiendo el mismo patrón mostrado en el gráfico general $(n=99)$.

Es curioso que las acciones «capacitar/ enseñar» se repitan más en Sename ( $\mathrm{n}=10)$, que en Integra $(n=2)$ y Junji $(n=4)$, organismos dedicados a la educación preescolar. Sin embargo estos últimos reemplazan dichos términos por «lograr». Ello indica que se enfocan en el resultado (medible, comparable), más que en el proceso de aprendizaje, ajustándose a los parámetros con los que se trabaja en educación actualmente en Chile, en los que cada establecimiento compite por obtener el mejor puntaje en la PSU (prueba de selección académica), o los mejores resultados en el Simce (Sistema de Medición de la Calidad de la Educación). En contraposición, la acción «proteger» tuvo mayor presencia en Junji $(n=8)$, que en Sename $(n=3)$, a pesar de que es una de sus labores. 
En tanto, la acción «fortalecer» está concentrada en Integra, Sename y Junji, instituciones que trabajan solo con niñas y niños, y las y los jóvenes en situación de vulnerabilidad ( $\mathrm{n}=73$ ). «Consultar», solo estuvo en una ocasión en el discurso de Integra $(n=1)$. Lo que corrobora la forma de trabajo de estas instituciones, que no consideran la opinión y/o planteamientos externos, ya sea por parte de niños, niñas y jóvenes, por parte de padres, madres y familiares, así como de otras organizaciones civiles.

\subsection{Acciones realizadas por el Estado por periodo de gobierno}

Al identificar diferencias y similitudes con respecto a cada gobierno, podemos establecer que, a nivel general, responden a los mismos lineamientos de gestión. En el mandato de Sebastián Piñera, hay mayor cantidad de acciones, las que se concentran en «realizar-hacer» $(n=77)$, «dar» $(n=51)$, «decir» $(\mathrm{n}=20)$, «enfocar» $(\mathrm{n}=13)$, «tener» $(\mathrm{n}=8)$, «aprobar» $(\mathrm{n}=13)$, «agrupar» $(\mathrm{n}=15), \mathrm{y}$ «lograr» $(n=23)$. Ello muestra que se trata de un discurso más centrado en la obtención de resultados.

No obstante, hay dos variables que se deben considerar: la primera, el terremoto que azotó a Chile en 2010 y la segunda, que se trata del primer gobierno de centro-derecha, elegido democráticamente, posterior a la dictadura. Por tanto, hay un «afán» por demostrar, pero también hay una mayor carga de trabajo luego de una catástrofe natural. Las acciones realizadas durante el primer gobierno de Michelle Bachelet, en mayor proporción son «fortalecer» $(n=40)$ y «actualizar» $(n=15)$. Ello está muy relacionado a las reformas ocasionadas por la Ley de Responsabilidad Penal Adolescente que se implementó en 2007.

Tabla 4. Acciones que realiza el Estado por periodo de gobierno

\begin{tabular}{|c|c|c|c|c|}
\hline \multicolumn{5}{|c|}{ Acciones que realiza el Estado } \\
\hline & & \multicolumn{2}{|c|}{ Periodo de gobierno } & \multirow{2}{*}{ Total } \\
\hline & & Piñera & Bachelet & \\
\hline \multirow{28}{*}{$\begin{array}{l}\text { Acciones que realiza el } \\
\text { Estado }\end{array}$} & Realizar, hacer & 77 & 64 & 141 \\
\hline & Capacitar, enseñar & 10 & 11 & 21 \\
\hline & Escoger, decidir & 4 & 0 & 4 \\
\hline & Fortalecer & 33 & 40 & 73 \\
\hline & Dar & 51 & 48 & 99 \\
\hline & Aumentar & 25 & 25 & 50 \\
\hline & Decir & 20 & 13 & 33 \\
\hline & Financiar & 3 & 4 & 7 \\
\hline & Mejorar & 24 & 21 & 45 \\
\hline & Homologar & 3 & 2 & 5 \\
\hline & Fiscalizar & 20 & 18 & 38 \\
\hline & Consultar & 1 & 0 & 1 \\
\hline & Identificar & 4 & 0 & 4 \\
\hline & Enfocar & 13 & 5 & 18 \\
\hline & Tener & 8 & 1 & 9 \\
\hline & Actualizar & 9 & 15 & 24 \\
\hline & Terminar & 6 & 1 & 7 \\
\hline & Aprobar & 13 & 3 & 16 \\
\hline & Agrupar & 15 & 8 & 23 \\
\hline & Interrumpir & 4 & 2 & 6 \\
\hline & Sensibilizar & 2 & 1 & 3 \\
\hline & Difundir & 19 & 14 & 33 \\
\hline & Proteger & 9 & 2 & 11 \\
\hline & Lograr & 23 & 16 & 39 \\
\hline & Atender & 4 & 7 & 11 \\
\hline & Atender & 3 & 1 & 4 \\
\hline & Utilizar & 2 & 0 & 2 \\
\hline & Otros & 2 & 0 & 2 \\
\hline \multicolumn{2}{|l|}{ Total } & 407 & 322 & 729 \\
\hline
\end{tabular}




\subsection{Estrategias discursivas}

Las estrategias discursivas utilizadas, entendidas como mecanismos que emplea el enunciador para resaltar determinados aspectos del discurso, sostienen un relato marcado por el pragmatismo. Las cuatro instituciones analizadas buscan principalmente cuantificar su quehacer. En este sentido, no se observan diferencias representativas entre los gobiernos de Michelle Bachelet y Sebastián Piñera. El uso de cifras es la clave cuando los textos tienen el fin de informar los logros de las instituciones analizadas, entregándolas como afirmaciones irrefutables, que le otorgan mayor credibilidad al discurso. Las estadísticas no son datos objetivos, son construcciones sociales que configuran determinadas visiones, tanto políticas como económicas, de la realidad y que incluso pueden ser sesgadas y tendenciosas, diseñadas para mostrar realidades disfrazadas (Best, 2009). Así la estrategia más utilizada ha sido la enumeración y el uso de cifras $(n=259)$, seguida de la descripción $(n=220)$. Debido al interés de las instituciones de mostrar un discurso neutral, la opinión ha sido una estrategia que apenas se ha utilizado en ninguno de los balances de gestión analizados $(n=1)$.

Gráfico 4. Estrategias discursivas

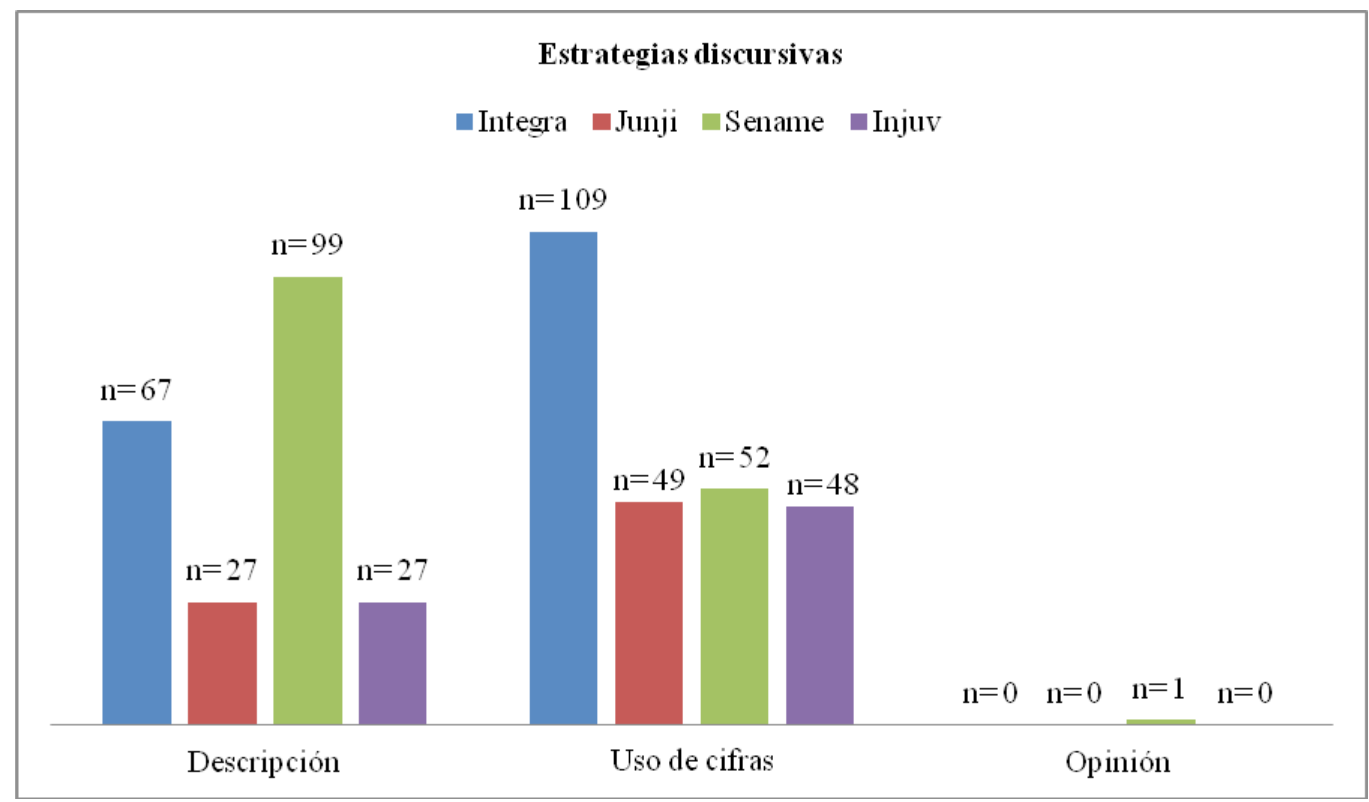

Las instituciones exhiben números favorables de la gestión presentándolos como mayoritarios, a pesar de que son casos aislados. Un ejemplo de aquello ocurre cuando el trabajo realizado en algunos de sus centros y/o jardines infantiles se presenta como una realidad nacional.

Otra tendencia muy habitual evidenciada en los textos analizados ha sido el uso de ejemplos particulares como si se tratasen de prácticas habituales o generalizadas. Dando así la sensación de que las instituciones tienen una praxis bien gestionada y organizada. Sename es una de las instituciones que más se vale de esta práctica, destacando los logros de los 10 centros que administra de forma directa como si representaran la totalidad de los establecimientos que brindan servicios cuando, en realidad, son muchos más.

«Otros logros relevantes fueron la implementación de talleres innovadores de arte y teatro, teniendo la máxima expresión en la puesta en escena de la obra vivencial 'Por un Minuto de Paz' por parte de seis adolescentes condenados del Centro Cerrado San Bernardo» [Balance de gestión, Servicio Nacional de Menores, Sename, 2008, p. 17]. 
Este tratamiento contrasta con la información publicada en El Mercurio el miércoles 5 de agosto con el título «El teatro ayuda a que los jóvenes del Sename eleven su autoestima». En el texto se especifica que solo se realizó un taller de teatro en el centro Tiempo Joven de San Bernardo, se explica cómo se trabajó con los jóvenes y los detalles de la presentación de la obra en la Fundación Telefónica.

«Desde agosto del año pasado, Roumeau asumió el desafío de trabajar con un grupo de chicos del centro Tiempo Joven, de San Bernardo. Es un centro cerrado por el que pasó, por ejemplo, el 'Poto rico', líder de la banda del 'Cisarro'. Ella trabaja con los chicos de la casa 3, que son los que están cumpliendo condena» (El Mercurio, 2009, párr. 3).

El uso de datos está relacionado directamente con las estrategias discursivas. Al clasificarlas en relación con los actos de habla, se puede notar que en la mayoría de los casos se producen actos asertivos, que como señala Searle (2001), corresponden a la representación de un determinado elemento como real o verdadero, en concordancia con el objetivo principal de estos escritos, donde se busca validar las acciones realizadas y los programas implementados a través de la enunciación de logros y metas cumplidas. Por ejemplo, con la proposición: «El 90,1\% de los jardines Junji obtuvieron logros significativos de aprendizaje» [Balance de Gestión, Junta Nacional de Jardines Infantiles, Junji, 2007, p. 59]. El uso de datos también va asociado al planteamiento desde una mirada positiva de los resultados de la gestión, siendo una característica compartida por ambos gobiernos.

Las instituciones no afirman directamente qué parte de una meta u objetivo no se cumplió, por el contrario, aluden a los esfuerzos que se realizaron, y ponen énfasis en los logros que se obtuvieron y, sobre todo, en pocas ocasiones utilizan la palabra «no». Para Van Dijk (2003, p. 158) esta es una estrategia básica de todo discurso ideológico que consiste en la autopresentación positiva, lo que se traduce para las instituciones en no hablar de los aspectos negativos de la organización y no hablar de los aspectos positivos de otras instituciones. Cuando las instituciones asumen que un objetivo no se logró, la justificación siempre corresponde a responsabilidades ajenas al organismo. Es decir que se enfatiza en los aspectos negativos de otros. Un ejemplo que retrata cómo una institución evade una meta no cumplida, es el extracto que forma parte de la cuenta pública de resultados en el Informe de gestión del Sename en 2006:

«En esta área es importante indicar que, si bien se realizaron todos los esfuerzos para limitar la permanencia de los niños y niñas internados en los centros residenciales, ya sea para su reinserción con su familia de origen o bien, para ubicarlo en una familia adoptiva, los resultados indican que en los tres centros que administra directamente Sename (Centro de Tránsito y Distribución de Lactantes y Preescolares), se logró disminuir desde 218 días a 155 días el tiempo promedio de permanencia de los niños y niñas egresados con familias. Sin embargo, este mismo indicador aplicado a los Centros que administran los Organismos Colaboradores aumentó en 9 días» [Informe de gestión, Sename, 2006, p. 23].

En la descripción precedente, Sename no habla de una meta no cumplida, sino que más bien solo da a conocer la información.

\subsection{Rol del Estado}

En cuanto al rol del estado, el análisis realizado arrojó los siguientes resultados. En primer lugar, la juventud es definida como un sujeto de consumo más que como uno de derechos. Injuv, organización que cambia de enfoque en el gobierno de Sebastián Piñera y que se centra en jóvenes en situación de pobreza, ofrece una mirada que también está presente en la construcción simbólica que realiza para definir sus programas. Esto se ve reflejado a través de la creación de una tarjeta destinada, 
en palabras del Estado, a «empoderarlos/las», dándoles acceso a descuentos en tiendas y reduciendo sus necesidades a un asunto económico. El término «empoderar», que es una palabra que por sí misma alude a un tipo de reivindicación social, es despojada de dicho significado, para ser utilizada como sinónimo de beneficios.

La plena realización se logra solamente cuando se respetan y garantizan las llamadas 'categorías' de derechos señaladas - Derechos Civiles y Políticos (DCP) y Derechos Económicos, Sociales y Culturales (Desc) - y garantizan la satisfacción de todos los derechos y no unos si y otros no, ya que los derechos humanos son en sí mismos el objetivo del desarrollo y del progreso y no un medio para alcanzarlo. Esta diferencia se aplica a los instrumentos de medición: los indicadores socioeconómicos miden el grado de desarrollo alcanzado, en cambio los indicadores de derecho miden si se ha logrado efectivamente el progreso a partir de la satisfacción de cada derecho (Pautassi \& Royo, 2012, p. 9).

Es decir, el Estado, en relación con juventud, cree cumplir su propósito facilitando el acceso a ciertos productos y servicios, que se podrían considerar como «derechos económicos», olvidando aquellos sociales y culturales, puesto que se centra las problemáticas juveniles en términos de acceso a bienes de consumo, en vez de ser una entidad que garantiza el cumplimiento de los derechos de las y los jóvenes, y contribuyendo, en conjunto con este grupo etario, en la creación de políticas y leyes.

Lo expuesto tiene relación con la asimetría social y la conceptualización que se hace de la infancia y la juventud subordinadas a la figura del adulto. La adjetivación de menor como contraparte del mayor (adulto), la «complitud» por sobre lo incompleto, la racionalidad versus la irracionalidad de la juventud e infancia son otros términos que han sido naturalizados. Bajo esta perspectiva, niñas y niños, y las y los jóvenes no son vistos como lo que son, sino por los sujetos que podrían llegar a ser (es decir que aún no son sujetos). Este imaginario adultocéntrico «constituye una matriz sociocultural que ordena naturalizando lo adulto como lo potente, valioso y con capacidad de decisión y control sobre los demás, situando (...) en condición de inferioridad y subordinación a la niñez, juventud y vejez» (Duarte, 2012, p. 120) y se mantiene por la concepción de que a pesar de tener una jurisprudencia que otorga derechos, el Estado todavía actúa bajo la lógica de la «necesidad» y la subordinación existente en la relación entre las personas adultas y niños, niñas y jóvenes (Figueroa-Grenett, 2018).

En segundo lugar, a raíz de las intervenciones sociales destacadas en los informes analizados es posible vislumbrar la unilateralidad de este organismo, que demuestra que el Estado se ve como único organismo facultado para hacerse cargo de la infancia y la juventud, ya que no les otorga herramientas a madres, padres o familiares. Todo lo contrario, les resta facultades en la toma de decisiones, al mismo tiempo que los excluye de sus intervenciones. En este sentido se asume que la familia, limitada económicamente, no solo carece de recursos sino también de competencias. En el caso específico de los y las jóvenes, son doblegados/as a una condición de meros/as consumidores/as, reemplazando a la figura del «padre proveedor» por la del «Estado proveedor», reforzando el sistema capitalista neoliberal. En esta lógica, Morlachetti (2013) repara en que el estado chileno no ha abandonado las características propias de un estado tutelar, lo cual imposibilita el reconocimiento universal de derechos.

El año 2014, se crea el Consejo de la Infancia definido como «instancia asesora presidencial que integra los esfuerzos de diversos organismos públicos, coordinando y dirigiendo sus acciones hacia el diseño y establecimiento de un sistema integral de garantías de los derechos de la infancia y la adolescencia, donde el Estado contempla su rol de garante», como una de las grandes promesas de programa de Michelle Bachelet. Del extracto se puede inferir que el Estado está consciente de algunas de sus falencias, como la desarticulación en el actuar de las diferentes instituciones, de la inexistencia del rol estatal como garantista en el cumplimiento de los derechos, y la falta de un sistema integral para infancia y juventud. La creación del Consejo de la Infancia, si bien tiene por objetivo respetar, promover y proteger el ejercicio de los derechos de niños, niñas y jóvenes, no se ha desprendido de las relaciones macroestructurales y de dominación que afectan a la infancia y la juventud. Lo anterior se refleja en su misión, que la explicitan con su deseo de «que todos los niños, niñas y adolescentes de Chile sientan que constituyen el bien más apreciado por sus familias, por su comunidad cercana, y para la sociedad en general» (Consejo de la Infancia, s. f., párr. 5). 
En esta declaración de principios, el consejo supone a niños, niñas y jóvenes como propiedad de sus familias, de la comunidad y la sociedad, lo que constituye un error si lo que se quiere es ver a este grupo como personas con capacidades y derechos para integrarse plenamente a la sociedad, y continúa remitiendo al patrón adultocéntrico que coarta libertades y vulnera facultades. Resulta paradójico que un organismo que debe reconocer, respetar, garantizar, promover y crear condiciones para adoptar derechos como la libertad, igualdad y dignidad de las personas, sin distinción de raza, color, sexo, idioma, religión, opinión política, origen nacional o social, posición económica, nacimiento, o cualquier otra condición, termine obviándolos y actuando contra su cumplimiento.

\section{Conclusiones y retos de futuro}

En relación con la primera pregunta de investigación planteada en este estudio - ¿cómo es el discurso del Estado chileno en torno a infancia y juventud? -, se puede concluir que Integra, Junji, Sename e Injuv centran su discurso en la difusión del trabajo realizado y la cobertura de sus programas o centros, relegando a un lugar secundario las implicancias de estos para las personas sobre las que recaen sus servicios: niñas y niños, y las y los jóvenes. Es más, sobre las personas menores de 18 años, las instituciones mantienen un discurso homogéneo en tres áreas fundamentales: participación, opinión y rol que desempeñan. Sin embargo, sí existen algunas diferencias entre una organización y otra, las cuales no son especialmente significativas.

Con respecto a la segunda pregunta planteada, sobre cuáles son los elementos clave que componen la propuesta discursiva de las instituciones que trabajan con infancia y juventud, se puede concluir lo siguiente: Sename discrimina a niños, niñas y jóvenes y, conjuntamente, hace lo mismo con sus familias. Ello se puede observar en sus objetivos estratégicos, los cuales están centrados exclusivamente en la propia institución; en la utilización de la palabra «menor/res», término que puede considerarse peyorativo; y en la ausencia de madres, padres y de la familia en general en sus informes. Junji, al igual que Sename, también discrimina e ignora a niñas y niños. De este modo, pareciera que sitúa sus objetivos estratégicos en la propia institución, sin considerar cómo los objetivos o propuestas podrían afectar a su público objetivo. Conjuntamente se refiere a los niños, niñas y jóvenes como «párvulo/os», término que alude a personas de corta edad, fáciles de engañar e ignorantes. Integra, por su parte, no recurre a ninguna de las prácticas anteriores, pero tampoco asigna a niñas y niños un espacio de participación real, dado que utiliza el vocablo al igual que todas las organizaciones analizadas como sinónimo de asistir o estar. Injuv tampoco confiere a los y las jóvenes espacios de expresión y participación que concluyan en la construcción conjunta de políticas o programas acordes a los intereses y necesidades de este grupo etario. Más bien les otorga un rol pasivo al interior de la institución y los reduce a «recibir» beneficios o a asistir a eventos organizados por el Instituto Nacional de la Juventud.

A la tercera pregunta diseñada: ¿Existen diferencias y similitudes en torno al discurso sobre infancia y juventud desarrollado por Junji, Integra, Sename e Injuv entre el gobierno de Michelle Bachelet y el de Sebastián Piñera?, se pudo concluir lo siguiente: el trabajo de estas cuatro instituciones no ha variado significativamente en los dos períodos analizados. Se podría hablar, por tanto, de un discurso que es constante en los dos gobiernos (marzo de 2006 a marzo de 2014). No obstante hay dos diferencias: la primera es el cambio de enfoque que se le dio a Injuv durante el gobierno de Sebastián Piñera. Esta modificación tuvo relación con orientar el trabajo de la institución hacia las personas en situación de vulnerabilidad, es decir, que con ello todas las organizaciones estatales que trabajan con infancia y juventud están dirigidas a niños, niñas y jóvenes en situación de vulnerabilidad. Esta orientación no otorga soluciones, es más, constituye una doble vulneración y estigmatización. Esto se debe a que asocia la niñez y la juventud con la pobreza, la delincuencia o el abuso, por ejemplo. La segunda diferencia es el enfoque de género presente durante el periodo de Michelle Bachelet, que se pudo advertir cuando las instituciones analizadas se referían a «niñas y niños» o «las y los jóvenes», 
expropiando a lo masculino de todo el espacio semántico y visibilizando al género femenino.

En consecuencia, al hablar del rol del Estado chileno, en el tratamiento mediático de la niñez y la juventud, no se pudieron establecer diferencias significativas entre los dos gobiernos analizados. La infancia está determinada por el acceso que se tenga a los programas de gobierno, dando por sentado que las niñas y niños que no necesitan de las instituciones de infancia, tienen todas sus necesidades cubiertas y, por ende, no deben ser asunto de consideración en el trabajo de Estado: por lo mismo, no existen. Padres, madres y/o apoderados también quedan exentos de esta construcción de significados, ya que el Estado, particularmente representado a través de Sename, los considera personas no aptas para el cuidado de niñas y niños, por tanto en vez de contribuir y ayudar para que desempeñen esta tarea en forma exitosa, impone modos de hacer y ver, coartando sus libertades y recalcando su visión paternalista hacia las y los ciudadanos, sobre todo aquellos con menores recursos económicos.

Las instituciones internacionales relacionadas con la infancia, tales como Unicef y organismos de carácter internacional como el Pnud, la Ocde o la ONU, entre otros, u organizaciones sociales nacionales que trabajan con niñas y niños, y con los y las jóvenes son también excluidas en la construcción e implementación de políticas y programas. Igualmente es posible observar la ausencia de una comunicación inter-institucional, tanto con organismos mayores, como el Ministerio de Educación, de Justicia y de Desarrollo Social, u otras entidades estatales que cumplen funciones similares o que trabajan con el mismo público. La ausencia de una visión, evaluación y trabajo en conjunto demuestra una comprensión incompleta porque las funciones con las que debe cumplir el Estado han sido desarrolladas para que cada una de las partes trabaje de acuerdo con su área técnica, a pesar de necesitar del complemento y esfuerzo de diferentes entidades. Como señalan Contreras et al. (2015), se necesita una política unitaria que articule el trabajo y tipo de intervención. Con el fin de evitar que primen los mandatos institucionales propios de cada sector.

En virtud de lo señalado, niñas y niños, y los y las jóvenes son utilizados como herramientas por el Estado para demostrar su «eficiencia» en esta materia. Sin embargo, no se denota un interés real por las personas que deberían ser el centro de sus políticas y su principal preocupación porque las niñas, niños y jóvenes no tienen participación en la construcción de políticas, planes, estrategias, proyectos, programas o talleres. A este respecto, no solo se identifica que no tenían injerencia, ni siquiera en los temas que les conciernen directamente, sino que tampoco opinión, obviando los artículos 12, 13, 14 y 15 de la Convención de los Derechos del Niño (y la niña).

Esta perspectiva tiene su arista social en una forma determinada de hacer políticas públicas: la promoción de la integración, que conlleva una visión específica de lo que es justicia e igualdad. Para Sandoval (2003), el discurso sobre integración se implementaría a través de la definición de la ciudadanía «desde la lógica de la redistribución de bienes y servicios de protección social, reorganización del trabajo, promoción de subsidios y de un sistema de políticas públicas centradas en la mejora de la desigualdad» (p.37). Estas políticas se materializan «en lo asistencial, paliativo y residual, obviándose la posibilidad de intervenir en el nivel de las estructuras que dan lugar a estas situaciones, y de aplicar políticas auténticamente preventivas...» (Gaitán, 2006, p. 76). Esta mirada está presente en tres de las cuatro instituciones públicas analizadas que fueron creadas para proteger los derechos de niños, niñas y jóvenes en situación de vulnerabilidad (Sename, Integra, Junji), por tanto el énfasis está en aquellos que no tienen recursos.

La principal problemática de esta forma de pensar las políticas públicas es que niños, niñas y jóvenes como grupo etario, independientemente del contexto socioeconómico en el que vivan, son vulnerados en sus derechos. Por tanto, la desigualdad estructural no radica particularmente en la pobreza. Por este motivo, el enfoque propuesto por el discurso estatal contribuye a acentuar las diferencias, hace visibles las realidades de ciertos grupos sociales y olvida que las carencias también se pueden presentar en otros contextos socioeconómicos.

La falta de recursos se consolida como el foco central de las agendas y es presentada como el 
problema principal que tienen niños, niñas y jóvenes, del que se desprenden otros. Como carencia afectiva, falta de educación, abandono, conductas de riesgo y un sinfín de otras variantes. Lo que da cuenta de «un problema cultural de construcción de las infancias marginadas, y a la vez, pone en cuestión la capacidad de quienes diseñan y ejecutan las políticas públicas a partir de estas construcciones» (Valdenegro \& Calderón-Flández, 2016, p. 1193). Si bien dicha relación existe, se invisibiliza que estas otras problemáticas pueden existir sin la variante económica de por medio. En otras palabras, un niño, una niña o un/una joven que goza de un contexto privilegiado puede igualmente encontrarse en una situación de abandono o desprotección de sus derechos, de la misma forma que infantes y jóvenes que provienen de un entorno de escasos recursos no necesariamente son víctimas de abusos, violencia u otro tipo de vulneración.

En cuanto al rol del Estado, este se caracteriza por su paternalismo y por ofrecer un punto de vista adultocéntrico que representa a la niñez y la juventud como una etapa de la vida carente de capacidades. Es más, el joven es representado en muchas instancias como un sujeto de consumo más que como uno de derechos. Además, se asume que la familia, limitada económicamente, no solo carece de recursos sino también de competencias, adoptando un tono paternalista y protector.

En este sentido, este artículo pretende ser un punto de partida para futuras investigaciones que aborden los discursos estatales y sus consecuencias. Justamente sería muy interesante preguntarse si la representación de infancia y juventud por parte del Estado chileno es compartida por otras naciones que han vivido procesos históricos similares, como los países del cono sur, y qué características diferenciadoras existe entre uno y otro, por ejemplo.

\section{Agradecimientos}

Agradecimientos a la Universidad de Santiago de Chile, concretamente, a la Escuela de Periodismo de la Facultad de Humanidades, que apoyó institucionalmente esta investigación.

\section{Lista de referencias}

Arias, B. (2017). La infancia como sujeto de derechos. Un análisis crítico. Ratio Juris, 12(24), 127-142. doi:10.24142/raju.v12n24a6

Berelson, B. (1971). Content analysis in communication research. Nueva York: Free Press.

Berger, P., \& Luckmann, T. (1968). La construcción social de la realidad. Buenos Aires: Amorrortu.

Best, J. (2009). Uso y Abuso de las estadísticas: la distorsión en la percepción pública de los problemas sociales y políticos. Santiago de Chile: Cuatro Vientos.

Casas, F. (2006). Infancia y representaciones sociales. Política y Sociedad, 43(1), 27-42. doi:10.5209/ POSO.23779

Consejo de la Infancia. (s. f.) Misión. Recuperado de: http://www.consejoinfancia.gob.cl/consejo/ que-es-el-consejo/\#mision

Contreras, J. I., Rojas, V., \& Contreras, L. (2015). Análisis de programas relacionados con la intervención en niños, niñas y adolescentes vulnerados en sus derechos: la realidad chilena. Psicoperspectivas, 14(1), 89-112. doi:10.5027/psicoperspectivas-Vol14-Issue1-fulltext-528

Del Río, E. (2011). Participación e intervención social con enfoque de derechos: análisis de un programa gubernamental. En O. Torres (Ed.) Niñez y ciudadanía, (pp. 78-107). Santiago de Chile: Pehuén.

Díaz-Bórquez, D., Contreras-Shats, N., \& Bozo-Carrillo, N. (2018). Participación infantil como aproximación a la democracia: desafíos de la experiencia chilena. Revista Latinoamericana de Ciencias Sociales, Niñez y Juventud, 16(1), 101-113. doi:10.11600/1692715x.16105

Duarte, C. (2012). Sociedades adultocéntricas: sobre sus orígenes y reproducción. Última Década, 20(36), 99-125. doi:10.4067/S0718-22362012000100005 
Durán-Strauch, E. (2017). Derechos de niños y niñas: del discurso a la política local. Revista Latinoamericana de Ciencias Sociales, Niñez y Juventud, 15(2), 879-891. doi:10.11600/169271 5x.1520623062016

Durkheim, E. (1975). Educación y sociología. Barcelona: Península.

El Mercurio. (2009, agosto 5). Emociones y rehabilitación: el teatro ayuda a que los jóvenes del Sename eleven su autoestima. Recuperado de: http://diario.elmercurio.com/detalle/index. asp?id $=\{1$ aab44ea-4e9e-4cbe-b92e-f3a51dbde788 $\}$

Figueroa-Grenett, C. (2018). La acción política de niños, niñas y jóvenes en Chile: cuerpos, performatividad y producción de subjetividad. Revista Latinoamericana de Ciencias Sociales, Niñez y Juventud, 16(1), 199-212. doi:10.11600/1692715x.16111

Gaitán, L. (2006). Sociología de la Infancia. Nuevas perspectivas. Madrid: Síntesis.

Giorgi, V. (2012). Entre el control tutelar y la producción de ciudadanía: aportes de la psicología comunitaria a las políticas de infancia. En J. Alfaro, A. Sánchez, \& A. Zambrano (Eds.) Psicología Comunitaria y Políticas Sociales: Reflexiones y Experiencias, (pp. 201-225). Buenos Aires: Paidós.

Grassi, E. (2003). Políticas y problemas sociales en la sociedad neoliberal. La otra década infame 1. Buenos Aires: Espacio.

Gülgönen, T. (2016). Participación infantil a nivel legal e institucional en México- ¿Ciudadanos y ciudadanas? Revista Latinoamericana de Ciencias Sociales, Niñez y Juventud, 14(1), 81-93. doi :10.11600/1692715x.1414081014

Hart, R. (1993). La participación de los niños: de la participación simbólica a la participación auténtica. Bogotá: Nueva Gente.

James, A., \& Prout, A. (1990). Constructing and reconstructing childhood: New directions in the sociological study of childhood. Oxford: Routledge.

Junta Nacional de Jardines Infantiles-Junji. (2007). Balance de gestión integral 2007. Recuperado de: http://www.dipres.gob.cl/597/articles-36895_doc_pdf.pdf

Lozano-Vicente, A. (2016). Los derechos del niño: cuestiones sobre su fundamentación. Revista Latinoamericana de Ciencias Sociales, Niñez y Juventud, 14(1), 67-79. doi:10.11600/169271 5x.1413290515

Marín, D. (2011). Notas para pensar la constitución de un campo discursivo. En F. Causiño, \& A. Foxley (Eds.) Políticas Públicas para la Infancia, (pp. 55-76). Santiago de Chile: Comisión Nacional Chilena de Cooperación con Unesco.

Misle, O., \& Pereira, F. (2003). Hacia un nuevo paradigma en la educación infantil y juvenil para la prevención y tratamiento del abuso sexual. Recuperado de: http://www.iin.oea.org/Cursos_a_ distancia/explotacion_sexual/Lectura3.NuevoParadigma. pdf

Morlachetti, A. (2013). Sistemas nacionales de protección integral de la infancia: fundamentos jurídicos y estado de aplicación en América Latina y el Caribe. Santiago de Chile: Comisión Económica para América Latina y el Caribe (Cepal).

Organización de las Naciones Unidas-ONU. (1989). Convención sobre los Derechos del Niño. Recuperado de: http://unicef.cl/web/convencion-sobre-los-derechos-del-nino/

Pautassi, L., \& Royo, L. (2012). Enfoque de derechos en las políticas de infancia: indicadores para su medición. Santiago de Chile: Comisión Económica para América Latina y el Caribe (Cepal).

Real Academia Española-RAE. (2001). Menor. Participar. Párvulo. En Diccionario de la lengua española ( $22^{\circ}$ ed.)

Recuperado de: http://dle.rae.es/?id=Ouc027t

Recuperado de: http://dle.rae.es/?id=S09ab8h

Recuperado de: http://dle.rae.es/?id=S1y8chE

República de Chile (2001). Ley de Prensa. Ley 19.733 sobre las libertades de opinión e información y ejercicio del periodismo. Santiago de Chile: Diario Oficial de la República de Chile.

República de Chile. (1967). Ley 16.618 de menores. Santiago de Chile: Diario Oficial de la República de Chile. 
Rice, P. (1997). Desarrollo humano: estudio del ciclo vital. Madrid: Prentice-Hall.

Rodríguez, I. (2007). Para una sociología de la infancia: aspectos teóricos y metodológicos. Madrid: Centro de Investigaciones Sociológicas.

Sandoval, J. (2003). Ciudadanía y juventud: el dilema entre la integración social y la diversidad cultural. Última Década, 11(19), 31-45. doi:10.4067/S0718-22362003000200003

Searle, J. (2001). Actos de habla. Madrid: Cátedra.

Servicio Nacional de Menores. (2006). Balance de Gestión Integral 2006. Recuperado de: http:// www.dipres.gob.cl/597/articles-36496_doc_pdf.pdf

Servicio Nacional de Menores-Sename. (2008). Balance de Gestión Integral 2008. Recuperado de: http://www.sename.cl/wsename/otros/gestion/BGI_2008_SENAME.pdf

Servicio Nacional de Menores. (2011). Balance de Gestión Integral 2011. Recuperado de: http:// www.sename.cl/wsename/otros/gestion/BGI_2011_SENAME.pdf

Tankard, J. (1991). Media frames: Approaches to Conceptualization and Measurement. Communication Theory and Methodology Division Association for Education in Journalism and Mass Communication Convention, Boston, U. S. A.

Valdenegro, B., \& Calderón-Flández, C. (2016). Alteridad, infancia y delincuencia: análisis discursivo desde programas sociales hacia infancias en Chile. Revista Latinoamericana de Ciencias Sociales, Niñez y Juventud, 14(2), 1191-1204. doi:https://dx.doi.org/10.11600/1692715x.14221070714

Valverde, F. (2008). Intervención social con la niñez: operacionalizando el enfoque de derechos. MAD, 3, 95-119. doi:10.5354/0718-0527.2008.31055

Van Dijk, T. (2003). La multidisciplinariedad del análisis crítico del discurso un alegato a favor de la diversidad. En R. Wodak, \& M. Meyer (Eds.) Métodos de análisis crítico del discurso, (pp. 143177). Barcelona: Gedisa.

Van Dijk, T. (2009). Discurso y poder. Barcelona: Gedisa.

Villagrasa, C. (2015). Derechos de la infancia y la adolescencia: hacia un sistema legal. Anales de la Cátedra Francisco Suárez, 49, 17-41. Recuperado de: http://revistaseug.ugr.es/index.php/acfs/ article/view/3276/3304 